\title{
Impact of Crystal Surface on Photoexcited States in Organic-Inorganic Perovskites
}

\author{
Susanne T. Birkhold, Eugen Zimmermann, Tom Kollek, Daniel Wurmbrand, \\ Sebastian Polarz, and Lukas Schmidt-Mende**
}

Despite their outstanding photovoltaic performance, organic-inorganic perovskite solar cells still face severe stability issues and limitations in their device dimension. Further development of perovskite solar cells therefore requires a deeper understanding of loss mechanisms, in particular, concerning the origin and impact of trap states. Here, different surface properties of submicrometer sized $\mathrm{CH}_{3} \mathrm{NH}_{3} \mathrm{Pbl}_{3}$ particles are studied as a model system by photoluminescence spectroscopy to investigate the impact of the perovskite crystal surface on photoexcited states. Comparison of single crystals with either isolating or electron-rich surface passivation indicates the presence of positively charged surface trap states that can be passivated in case of the latter. These surface trap states cause enhanced nonradiative recombination at room temperature, which is a loss mechanism for solar cell performance. In the orthorhombic phase, the origin of multiple emission peaks is identified as the recombination of free and bound excitons, whose population ratio critically depends on trap state properties. The dynamics of exciton trapping at $50 \mathrm{~K}$ are observed on a time-scale of tens of picoseconds by a simultaneous population decrease and increase of free and bound excitons, respectively. These results emphasize the potential of surface passivation to further improve the performance of perovskite solar cells.

development of perovskite solar cells still faces several issues. These include the incorporation of toxic lead, insufficient device stability, as well as limitations in device area and thickness for high performance solar cells. ${ }^{[6-8]}$ Improvements in device dimensions require a deeper understanding of the fundamental light-induced processes and the corresponding loss mechanisms within the solar cell, in particular concerning the origin and impact of trap states. While shallow trap states, which are in the vicinity of conduction or valence band, lead to reduced charge carrier mobility, deep trap states can act as nonradiative recombination centers. ${ }^{[9]}$ In case of perovskite solar cells, it has been shown that nonradiative Shockley-ReadHall recombination caused by trap states is the dominant recombination mechanism under standard illumination intensities. ${ }^{[10,11]}$ Following considerations of detailed balance, this nonradiative recombination is a general loss mechanism for solar cell performance, ${ }^{[12,13]}$ which

\section{Introduction}

Hybrid organic-inorganic perovskites have attracted rapidly increasing interest as material for solar energy conversion, as they are the first class of solution-processable semiconductors that can lead to solar cell efficiencies comparable to crystalline silicon solar cells. ${ }^{[1]}$ From 2009 to 2015, the energy conversion efficiency of perovskite solar cells could be raised from $3.8 \%$ to over $20 \%$ by optimizing film deposition techniques, device architectures, and material compositions. ${ }^{[2-5]}$ Despite the tremendous achievements in device performance, further

\footnotetext{
S. T. Birkhold, E. Zimmermann,

Prof. L. Schmidt-Mende

Department of Physics

University of Konstanz

Universitätsstr. 10, 78457 Konstanz, Germany

E-mail: Lukas.schmidt-mende@uni-konstanz.de

T. Kollek, D. Wurmbrand, Prof. S. Polarz

Department of Chemistry

University of Konstanz

Universitätsstr. 10, 78457 Konstanz, Germany
}

has been confirmed by a decrease in $V_{\text {oc }}$ for perovskite solar cells. ${ }^{14,15]}$ Although $\mathrm{CH}_{3} \mathrm{NH}_{3} \mathrm{PbI}_{3}$ and $\mathrm{CH}_{3} \mathrm{NH}_{3} \mathrm{PbBr}_{3}$ single crystals prove the material's potential of extremely low trap state densities in the order of $10^{9}-10^{10} \mathrm{~cm}^{-3},{ }^{[16]}$ resulting from their defect-tolerant transition orbital configuration, ${ }^{[17]}$ trap state densities are raised to $10^{16}-10^{17} \mathrm{~cm}^{-3}$ for commonly processed films of $\mathrm{CH}_{3} \mathrm{NH}_{3} \mathrm{PbI}_{3-x} \mathrm{Cl}_{x}$ and $\mathrm{CH}_{3} \mathrm{NH}_{3} \mathrm{PbI}_{3} \cdot{ }^{[11,18]}$ The intimate relation between trap state properties and solar cell performance underlines the importance to better understand the defect physics of organic-inorganic perovskites and to develop methods for trap state passivation.

Based on density functional theory (DFT) simulations, point defects like lead and iodide interstitials, antisites, or vacancies have been proposed to cause both shallow and deep trap states within the crystal bulk. ${ }^{[9,19]}$ The existence of deep trap states in perovskite solar cells has been experimentally proven by thermally stimulated current measurements, revealing trap state energies as large as $500 \mathrm{meV}^{[20]}$ Further indications for the formation of iodide-related point defects are given by the dependence of the carrier diffusion length on iodide-poor growth conditions, as for mixed halides $\mathrm{CH}_{3} \mathrm{NH}_{3} \mathrm{PbI}_{3-x} \mathrm{Cl}_{x}$ and $\mathrm{Pb}(\mathrm{Ac})_{2}$ precursors..$^{[9,21]}$

While theoretical and experimental results are in good agreement concerning the origin of bulk trap states, only a limited 
number of publications investigated the impact of crystal surfaces and grain boundaries on trap state formation in organicinorganic perovskites. Although DFT simulations predict bulklike delocalized states at crystal surfaces and shallow trap states at grain boundaries, ${ }^{[9,22,23]}$ experimental findings lead to contradictory conclusions. In case of $\mathrm{CH}_{3} \mathrm{NH}_{3} \mathrm{PbI}_{3}$ films, scanning Kelvin probe measurements reveals only small potential differences of around $15 \mathrm{meV}$ between grains and grain boundaries, ${ }^{[24]}$ while photoluminescence (PL) maps show a PL intensity drop of $65 \%$ at grain boundaries, associated with an increased density of deep trap states. ${ }^{[25]}$ However, also for single crystals of $\mathrm{CH}_{3} \mathrm{NH}_{3} \mathrm{PbI}_{3}$ the presence of trap states at plain crystal surfaces has been suggested, as Kelvin probe measurements reveal a Fermi level shift of $120 \mathrm{meV}$ at the crystal surface compared to the bulk. ${ }^{[26]}$ Likewise, improved solar cell performance and reduced hysteresis by surface passivation of perovskite films with electron-rich molecules like thiophene, pyridine, or [6,6]-phenyl- $\mathrm{C}_{61}$-butyric acid methyl ester (PCBM) derivatives highlight the presence of charged surface defects. ${ }^{[27-29]}$ Although these experimental findings indicate the presence of surface trap states for organic-inorganic perovskites, many questions related to their exact nature and their interaction with photoexcited states remain unclear.

In this work, we aim to contribute to a better understanding of the impact of the $\mathrm{CH}_{3} \mathrm{NH}_{3} \mathrm{PbI}_{3}$ crystal surface on the photoexcitation landscape and the dynamics of photoexcited states. As a model system for surface effects, we investigate submicrometer sized $\mathrm{CH}_{3} \mathrm{NH}_{3} \mathrm{PbI}_{3}$ single crystals with different surface passivation. ${ }^{[30]}$ Intensity- and time-resolved photoluminescence measurements are performed at different temperatures to study the impact of surface states on free charge carriers at room temperature, as well as on Wannier-Mott excitons at low temperatures. ${ }^{[31,32]}$ We identify charged surface trap states leading to increased nonradiative recombination rates and reduced PL lifetimes at room temperature and to bound excitons at low temperatures. Using time-resolved photoluminescence spectroscopy, we observe the trapping process of free excitons, forming bound excitons on a time-scale of tens of picoseconds. A comparison of surface passivation molecules based on either electron-rich hexylthiophenes or isolating alkyl chain reveals reduced trap state densities and energies in case of surface modification with hexylthiophene, indicating the presence of positively charged defects at the crystal surface.

\section{Results and Discussion}

\subsection{Surface Modified $\mathrm{CH}_{3} \mathrm{NH}_{3} \mathrm{Pbl}_{3}$ Crystals}

Single-crystalline $\mathrm{CH}_{3} \mathrm{NH}_{3} \mathrm{PbI}_{3}$ cuboids with dimensions of around 500-1000 nm (see Figure 1 and Figure S1, Supporting Information) were synthesized using a particle growth method based on the single source precursor $\mathrm{CH}_{3} \mathrm{NH}_{3} \mathrm{PbI}_{3} \mathrm{TEG}_{2}$ as described in Section 4. ${ }^{[30]}$ A high surface-to-volume ratio due to their submicrometer size, as well as a low bulk trap state density makes these single crystals an ideal model system to study the impact of the crystal surface on photoexcited states. The crystals are surface passivated by organic molecules that
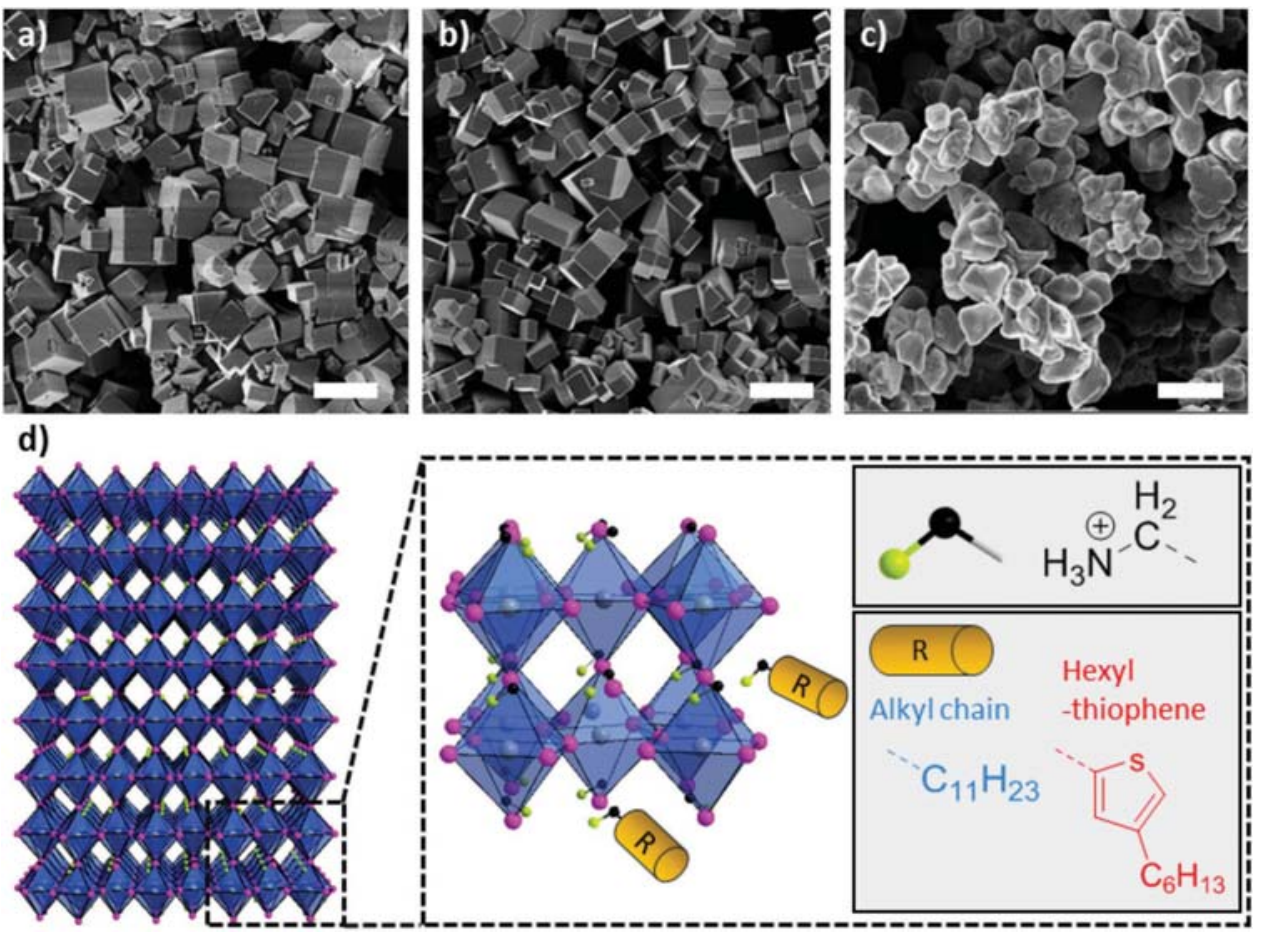

Figure 1. SEM images of a) alkyl chain cuboids, b) hexylthiophene cuboids, and c) unpassivated particles. Scale bar is $2 \mu \mathrm{m}$. d) Schematic illustration of surface passivation of $\mathrm{CH}_{3} \mathrm{NH}_{3} \mathrm{Pbl}_{3}$ cuboids ( $=$ pink, $\mathrm{Pb}=$ grey, $\mathrm{N}=$ green, $\mathrm{C}=$ black). Right: Surface molecules are composed of a $\mathrm{NH}_{3}{ }^{+}$head group (top) and a functional end group "R" (bottom). Broken lines indicate chemical bonds between $\mathrm{NH}_{3}{ }^{+}$head and functional end group. The $\mathrm{NH}_{3}{ }^{+}$head group attaches to the crystal surface at the $A$ site of the $A B X_{3}$ structure. 
consist of a $\mathrm{NH}_{3}{ }^{+}$head group and a functional end group. During particle growth, these passivation molecules attach to the A site of the $\mathrm{ABX}_{3}$ perovskite structure by ionic interactions and hydrogen bonding of the $\mathrm{NH}_{3}{ }^{+}$head group. ${ }^{[3,34]}$ To evaluate the nature of surface states and the effectiveness of their passivation, two different functional end groups are applied, which are either formed by an isolating alkyl chain $\left(\mathrm{C}_{12} \mathrm{H}_{25}\right)$ or by a conjugated hexylthiophene $\left(\mathrm{C}_{11} \mathrm{H}_{17} \mathrm{~S}\right)$ possessing a high electron density at its sulfur atom. These cuboids will be called alkyl chain cuboids and hexylthiophene cuboids in the following. For their surface passivation, the surfactants dodecylammonium iodide and (4-hexylthiophen2-yl)methylammonium iodide were applied during particle growth, respectively. As the surface molecules not only vary in their electronic properties, but also in their steric structure, different attachment modes of the molecules to the crystal surface are observed. Scanning electron microscopy (SEM) images displayed in Figure 1 and Figure S1 in the Supporting Information reveal slightly rod-liked structures for some hexylthiophene cuboids that deviate from the highly cubic structures of the alkyl chain cuboids. Likewise, X-ray diffraction (XRD) measurements presented in Figure S2 (Supporting Information) indicate a small variation in the aspect ratio of crystal directions caused by the different surface modifications. These differences can be explained by the bulky size of the hexylthiophene causing a favored linkage to the [100] and [010] crystal direction of the perovskite crystal, due to the tetragonal distortion that leads to a larger opening angle of the stretched inorganic octahedron at this crystal direction. ${ }^{[35]}$ In contrast, the straight alkyl chain is well known to have a favorite attachment in the [001] crystal direction. This facet-selective attachment of linker molecules to different $\mathrm{CH}_{3} \mathrm{NH}_{3} \mathrm{PbI}_{3}$ crystal directions and its impact on the crystalline shape have already been reported for similar submicrometer sized particles passivated by alkyl chains and will be discussed in detail for surface passivation with hexylthiophene elsewhere. ${ }^{[30]}$ The different steric demands of the passivation molecules also influence the surface coverage of functionalization, as NMR studies of dissolved cuboids reveal a reduction in surface coverage for hexylthiophene cuboids by a factor of 10 compared to the alkyl chain cuboids (see Figure S3, Supporting Information). This dual influence of surface passivation on both the electronic and crystallographic surface landscape will be considered in the following study.

Additionally, particles without a selective surface passivation (preparation described in Section 4) are included in the study as well, which were synthesized according to the same procedure but without additional surface ligands. These particles will be called unpassivated particles in the following. The absence of surface passivation molecules during crystal growth results in poly-crystalline particles with uncontrolled geometry. This comparison of unpassivated particles to passivated cuboids allows to compare intrinsic and modified crystal surfaces.

\subsection{Room Temperature Photoluminescence}

Figure 2 shows the absorbance and PL measured at $295 \mathrm{~K}$, which we account to the radiative band-to-band transition of electrons and holes. Although the exact nature of initially excited states in organic-inorganic perovskites is still under debate, ${ }^{[31,36]}$ recent reports support the notion of free charge carriers at room temperature and dominant Wannier-Mott type excitons at low temperatures based on exciton binding energies determined to be below $10 \mathrm{meV}$ at room temperature and $16-30 \mathrm{meV}$ at low temperatures. ${ }^{[37-39]}$ Figure 2a displays relatively similar absorbance and PL spectra at $295 \mathrm{~K}$ for the different particles. However, the PL decays presented in Figure $2 \mathrm{~b}$ show an increased decay rate for the unpassivated particles. This reduced PL lifetime is caused by increased nonradiative trap state recombination due to a higher trap state density either in the bulk of the polycrystalline particles or at their crystal surface. ${ }^{[11]}$ However, the PL lifetime also differs between the different surface passivation molecules of the single-crystalline cuboids, with a reduced PL lifetime in case of isolating surface passivation. As the alkyl chain cuboids and the hexylthiophene cuboids only differ in their surface properties, a higher density of surface trap states can be concluded for the alkyl
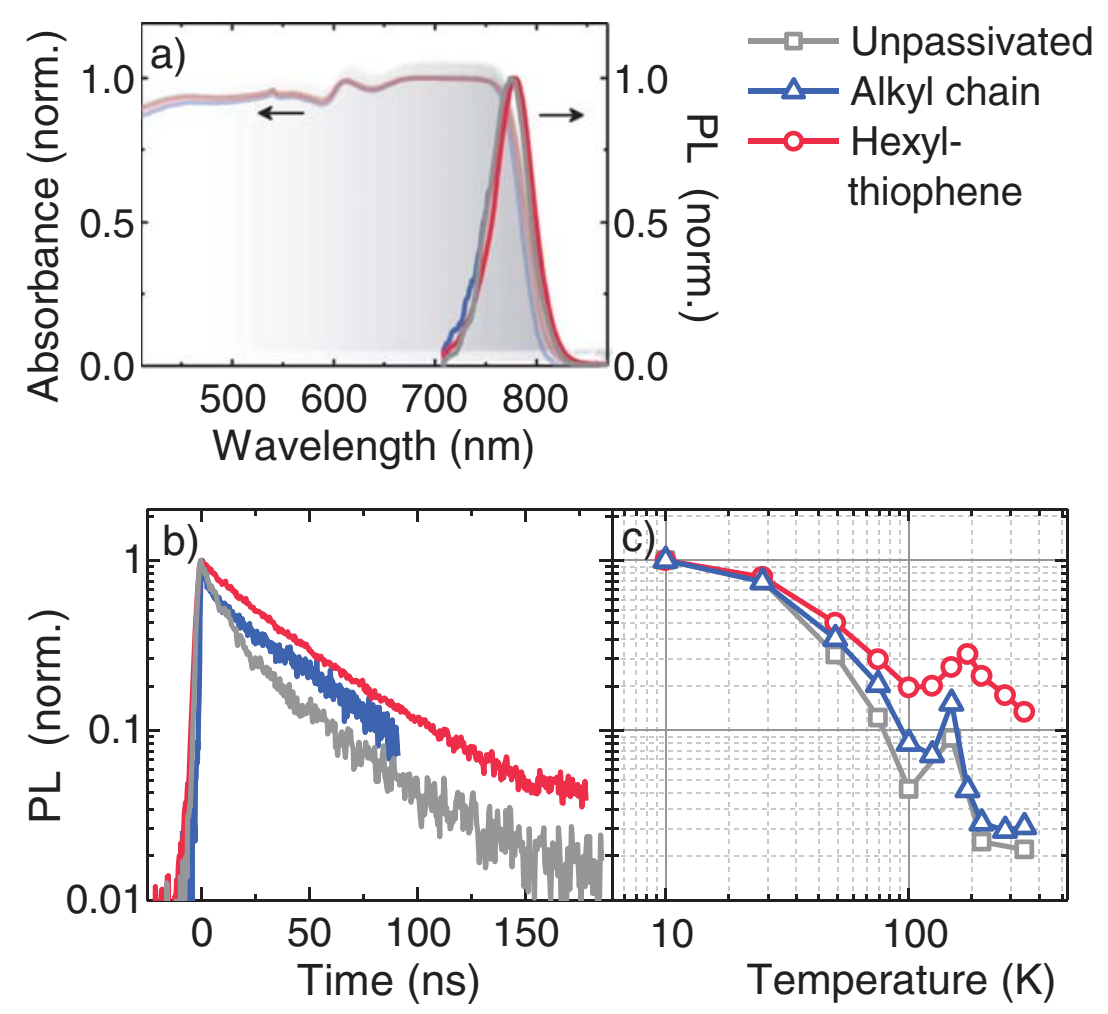

Figure 2. Absorbance and $\mathrm{PL}$ of $\mathrm{CH}_{3} \mathrm{NH}_{3} \mathrm{Pbl}_{3}$ particles. a) Absorbance and $\mathrm{PL}$ spectra at $295 \mathrm{~K}$, b) PL decay at $295 \mathrm{~K}$, and c) integrated PL intensity as a function of temperature, normalized to the PL intensity at $10 \mathrm{~K}$. 
chain cuboids, leading to enhanced nonradiative recombination and shorter PL lifetimes.

The varying impact of passivation molecules on surface states is further supported by the dependency of the total PL intensity on temperature, displayed in Figure 2c. Here, thermally activated quenching processes reduce the PL intensity for organic-inorganic perovskites with increasing temperature. ${ }^{[40,41]}$ The relation between the PL intensityIon temperature can be described as follows ${ }^{[42]}$

$$
I(T)=\frac{I_{0}}{1+A \exp \left(\frac{-E_{\mathrm{a}}}{k_{\mathrm{B}} T}\right)+B \exp \left(\frac{-E_{\mathrm{b}}}{k_{\mathrm{B}} T}\right)}
$$

with a PL intensity $I_{0}$ at zero temperature, an activation energy $E_{\mathrm{a}}$ for thermally induced exciton dissociation, and an activation energy $E_{\mathrm{b}}$ for nonradiative recombination processes caused by electron-phonon interactions. Assuming an efficiency of $100 \%$ for radiative emission at $10 \mathrm{~K}$, the PL intensity decreases from 10 to $295 \mathrm{~K}$ to $1.9 \%$ and $2.7 \%$ for the unpassivated particles and the alkyl chain cuboids, respectively. In case of the hexylthiophene cuboids, thermally induced nonradiative recombination pathways are significantly reduced, leading to a noticeably higher radiative efficiency of $12.8 \%$ at $295 \mathrm{~K}$. As we do not assume a significant change in the exciton binding energy $E_{\mathrm{a}}$ for the different particles, these differences in PL intensity are more likely due to variations in the activation energy $E_{\mathrm{b}}$ for interactions between photoexcited states and phonons. Based on the significant decrease in nonradiative recombination pathways compared to the alkyl chain cuboids, this indicates reduced lattice interaction and hence less surface lattice distortions for the hexylthiophene cuboids.

We want to note that the peak in PL intensity around the phase transition at $165 \mathrm{~K}$ occurs reproducibly for all particles (Figure 2c). Similar amplification of the PL emission around the orthorhombic-tetragonal phase transition has also been reported for $\mathrm{CH}_{3} \mathrm{NH}_{3} \mathrm{PbI}_{3}$ films, yet its physical origin, e.g., a coexistence of the two phases, is still under debate. ${ }^{[43-45]}$

\subsection{Low Temperature Photoluminescence Spectra}

The PL spectra at temperatures below $100 \mathrm{~K}$ possess multiple emission peaks for all particles (see Figure 3a-d), whereas two different mechanisms have been reported for their physical

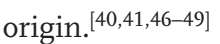

In both proposed mechanisms the high energy emission peak is addressed to free excitons (FEs) in the orthogonal phase. While one mechanism assigns the lower energy peak to remaining inclusions of the tetragonal phase with a narrower bandgap compared to the orthorhombic phase, ${ }^{[36,41,46-48]}$ the other mechanism describes the formation of bound excitons (BEs) that are localized at trap states. ${ }^{[40,49]}$ Phuong et al. assume that tetragonal inclusions are caused by strain imposed on grain boundaries in polycrystalline $\mathrm{CH}_{3} \mathrm{NH}_{3} \mathrm{PbI}_{3}$ films. It is therefore followed that this two-phase coexistence is not apparent in $\mathrm{CH}_{3} \mathrm{NH}_{3} \mathrm{PbI}_{3}$ single crystals. ${ }^{[36]}$ As the $\mathrm{CH}_{3} \mathrm{NH}_{3} \mathrm{PbI}_{3}$ cuboids investigated here do not exhibit grain boundaries, we assign the occurrence of multiple emission
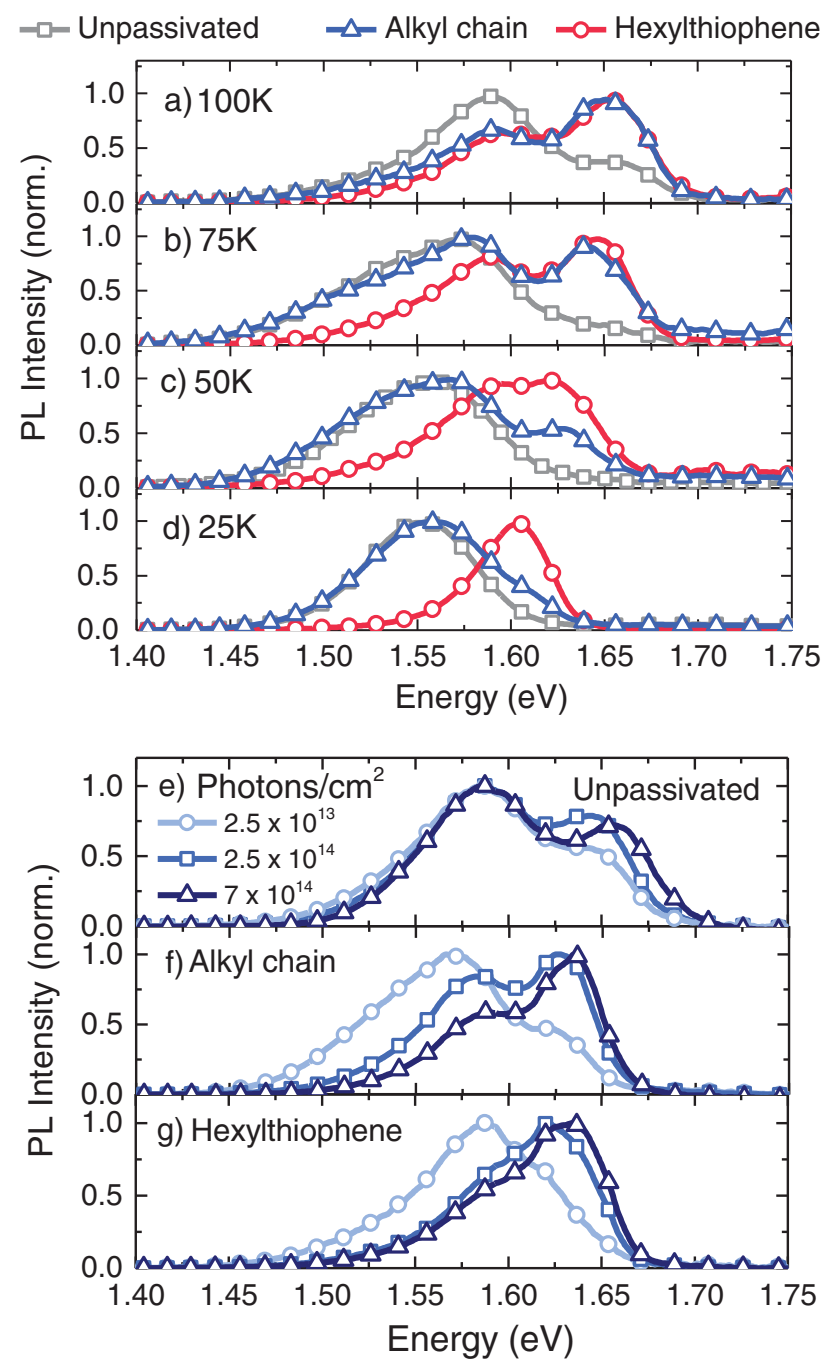

Figure 3. $\mathrm{PL}$ spectra of $\mathrm{CH}_{3} \mathrm{NH}_{3} \mathrm{Pbl}_{3}$ particles at temperatures below 100 K. a-d) PL spectra at different temperatures of 100-25 K. e-g) PL spectra at $50 \mathrm{~K}$ for different excitation intensities.

peaks to $\mathrm{FE}$ and $\mathrm{BE}$ according to the study by Fang et al. on $\left(\mathrm{CH}_{3} \mathrm{NH}_{3}\right) \mathrm{PbI}_{3}$ single crystals. ${ }^{[40]}$ The formation of bound excitons at temperatures below $100 \mathrm{~K}$ is supported by significant variation in the PL peak ratio for the different particles in Figure $3 \mathrm{a}-\mathrm{d}$. Especially in case of the alkyl chain and hexylthiophene cuboids, which only differ in their surface properties, we do not expect differences in the phase transition process, but rather in surface trap state density. Furthermore, we want to note that the lower energy PL peak of the particles studied here increases with decreasing temperature, which opposes the decrease of tetragonal inclusions with decreasing temperature observed for $\mathrm{CH}_{3} \mathrm{NH}_{3} \mathrm{PbI}_{3}$ films. ${ }^{[50]}$

For inorganic semiconductors like doped silicon, PL from BE has already been studied extensively. ${ }^{[51]}$ Thereby, excitons lose their kinetic energy owing to localization at trap states and the binding energy of the resulting BE equals the energy difference between the PL emission peaks of FE and BE. 
Compared to the single-crystalline cuboids, the unpassivated particles have a more pronounced lower energy peak for all temperatures between 25 and $100 \mathrm{~K}$, revealing an increased number of BE. In case of the cuboids, both PL spectra show similar and overall higher ratios of FE to BE at $100 \mathrm{~K}$. With decreasing temperature, however, the $\mathrm{BE}$ peak of the alkyl chain cuboids constantly increases and the PL spectrum approaches the spectrum of the unpassivated particles at $25 \mathrm{~K}$. For better comparison, the PL spectra were fitted with three Gaussian curves, one for the high energy peak FE and two for the lower energy peak of BE (BE1 and BE2), as exemplarily presented in Figure S4 (Supporting Information). The origin of the two lower energy peaks as trap state mediated BE has been clarified by Fang et al. by excitation intensity dependent PL measurements. ${ }^{[40]}$

The evolution of the peak ratio $\mathrm{FE} /(\mathrm{BE} 1+\mathrm{BE} 2)$ as a function of temperature is shown in Figure S5a (Supporting Information). A decrease in temperature leads to an increased trapping of excitons and formation of $\mathrm{BE}$ due to lacking energy of the surrounding thermal bath. Hence, the relation between BE population and temperature depends on the density and the energetic distribution of trap states.

To evaluate differences in the trap state density, excitation intensity dependent PL measurements are performed. Due to the limited number of trap states, an increase in excitation intensity will lead to a filling of trap states and an increasing amount of free photoexcited states upon a threshold intensity. As shown in Figure $3 \mathrm{~d}-\mathrm{f}$, an increase in excitation intensity only leads to a small increase of FE for the unpassivated particles, which supports their higher trap state density discussed above. In case of the single-crystalline cuboids, the same intensity increase causes a significantly stronger rise of the FE peak, leading to a continuous increase of the $\mathrm{FE} /(\mathrm{BE} 1+\mathrm{BE} 2)$ peak ratio with increasing excitation intensity for the alkyl chain cuboids, and a quick saturation for the hexylthiophene cuboids already at low excitation intensities. This intensity saturation effect reveals a further reduced trap state density for surface passivation with hexylthiophene compared to modification with isolating alkyl chains. We want to note that all excitation intensity dependent effects were instantaneous and reversible.

To study differences in the energetic distribution of trap states, the temperature evolution of the PL peak position is evaluated (see Figure S5b, Supporting Information). For all particles, similar FE peak positions prove the independency of FE energy from the surrounding trap state environment. We therefore conclude that FEs are mobile and gain additional energy with higher temperature due to interaction with the surrounding thermal bath. In case of unpassivated particles and alkyl chain cuboids, the higher energy peak BE1 features a similar energy increase with increasing temperature and approaches the energetically higher BE1 peak position of the hexylthiophene cuboids only at $100 \mathrm{~K}$. At $25 \mathrm{~K}$, the BE1 peak is blueshifted by $40 \mathrm{meV}$. The lower energy peak BE2, however, is for all particles rather independent of temperature between 25 and $100 \mathrm{~K}$, reflecting highly immobile and deeply trapped excitons. Again, for the hexylthiophene cuboids the BE2 peak position is blueshifted by $28 \mathrm{meV}$ at $25 \mathrm{~K}$. The blueshifted PL peaks for hexylthiophene surface passivation indicate reduced $\mathrm{BE}$ binding energies and likewise reduced trap state energies. For alkyl chain cuboids and unpassivated particles, on the other hand, similar trap state energies can be concluded due to their identical PL peak positions. The similarity in trap state energy of alkyl chain cuboids and unpassivated particles, in combination with the lower trap state density of the alkyl chain cuboids compared to the unpassivated particles, agree well with the temperature evolution of the PL spectra presented in Figure 3a-d. Based on the analogous trap state energies, the same nature of trap states can be assumed for none and isolating surface passivation. By contrast, the reduced density and energy of these surface trap states for hexylthiophene cuboids confirm the superior trap state passivation by hexylthiophenes. These findings are consistent with our measurements at room temperature and indicate a correlation of trap state properties between tetragonal and orthorhombic crystal phases.

\subsection{Low Temperature Photoluminescence Decays}

Time-resolved PL measurements are evaluated to study exciton dynamics and interactions between free and bound excitons at low temperatures. Figure $4 \mathrm{a}-\mathrm{c}$ shows PL decays at $10 \mathrm{~K}$ for the investigated particles. The PL dynamics can be fitted with biexponential decay functions, with a fast component of several hundreds

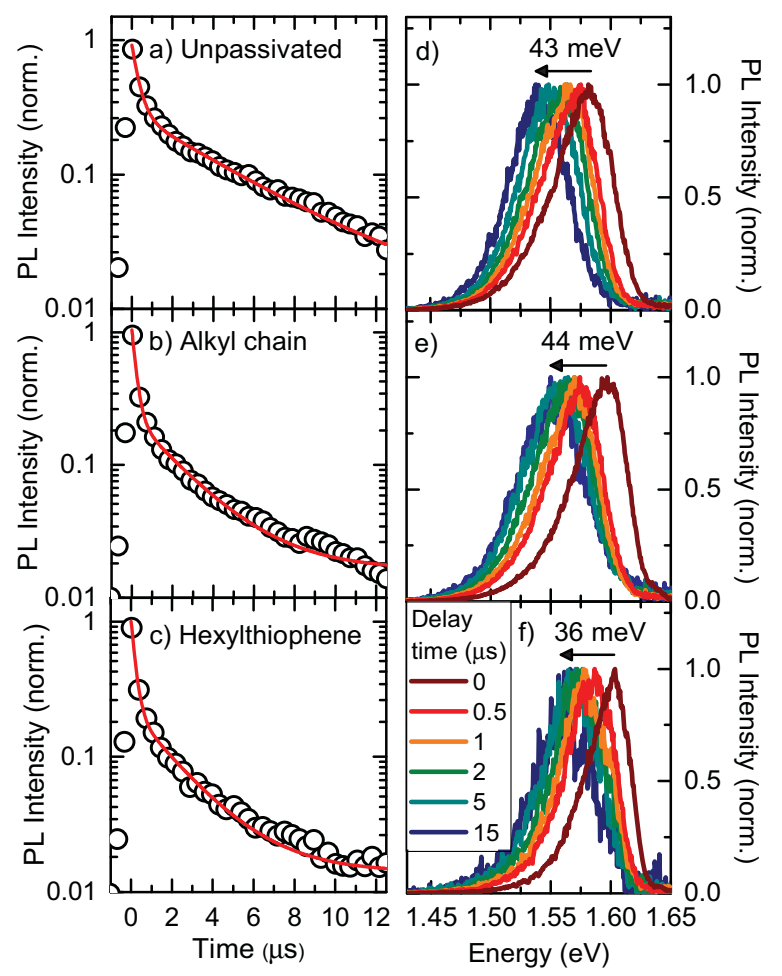

Figure 4. $\mathrm{PL}$ decays of $\mathrm{CH}_{3} \mathrm{NH}_{3} \mathrm{Pbl}_{3}$ particles at $10 \mathrm{~K}$. a-c) $\mathrm{PL}$ decays (black cycles) fitted with biexponential decays (red line). Fit results: Unpassivated: $t_{1}=0.36 \mu \mathrm{s}(41.6 \%)$ and $t_{2}=4.65 \mu \mathrm{s}(58.4 \%)$, alkyl chain cuboids: $t_{1}=0.21 \mu \mathrm{s}(85.4 \%)$ and $t_{2}=2.47 \mu \mathrm{s}$ (14.6\%), hexylthiophene cuboids: $t_{1}=0.21 \mu \mathrm{s}(85.7 \%)$ and $\left.t_{2}=2.29 \mu \mathrm{s}(14.3 \%)\right)$. Normalized $\mathrm{PL}$ spectra of d) unpassivated particles, e) alkyl chain cuboids, and f) hexylthiophene cuboids at different delay times after excitation. 
of nanoseconds and a slow component of several microseconds. While for the unpassivated particles the slow decay component dominates with a lifetime of $4.6 \mu \mathrm{s}$, the majority of photoexcited states in the single-crystalline cuboids recombines within the fast decay component with a lifetime of around $200 \mathrm{~ns}$.

Figure $4 \mathrm{~d}-\mathrm{f}$ presents the PL spectra detected at different delay times after photoexcitation. Initial states corresponding to the fast decay component are of higher energy, followed by continuously redshifting states with significantly longer lifetimes. Due to the differences in peak position and lifetime, we assign the initially emitting high energy states to FE and the long-lived, lower energy states to BE. Consistent to the PL spectra presented in Figure 3, the relative population of $\mathrm{BE}$ is higher for the unpassivated particles, while for the cuboids fast decaying FEs are more pronounced. The dynamic interaction between FE and BE will be analyzed in more detail below (Figure 5). Similar long PL lifetimes of BE of up to $5 \mu$ s have already been observed for $\mathrm{CH}_{3} \mathrm{NH}_{3} \mathrm{PbI}_{3}$ single-crystals at $5 \mathrm{~K}$ and have been attributed to bound triplet excitons. ${ }^{[40]}$ Apart from that, the significant difference in lifetime of $\mathrm{FE}$ and $\mathrm{BE}$ can be related to an efficient capturing of $\mathrm{FE}$ due to large radii of free Wannier-Mott excitons and resulting large capture cross section of trap states. Furthermore, a "giant oscillator strength" has been characterized for BE in inorganic semiconductors, ${ }^{[52]}$ which might also contribute to efficient luminescence of $\mathrm{BE}$ in organic-inorganic perovskites.

An energy shift of around $40 \mathrm{meV}$ takes place during the PL decay for all particles, which displays the energy difference of free and bound excitons at $10 \mathrm{~K}$. However, the dynamics of this temporal PL peak shift differ between the different particles. In case of the cuboids the major part of the PL shift takes place within the first $500 \mathrm{~ns}$, while for the unpassivated particles the energy shift evolves continuously during the whole PL lifetime of $>15 \mu \mathrm{s}$. This continuous redshift of the PL spectra reveals that excitons in the unpassivated particles interact with a broader energy distribution of trap states, while for the singlecrystalline cuboids excitons are affected by a smaller distribution of trap states. We therefore assign the single-crystalline cuboids a spatial restriction of trap states to the crystal surface and a high crystallinity within the crystal bulk.

To investigate the initial decay dynamics in more detail, timeresolved PL measurements are performed with enhanced time resolution on a smaller time range of 2 ns (Figure 5). These measurements are performed at $50 \mathrm{~K}$, where the $\mathrm{FE}$ and $\mathrm{BE}$ PL peaks are more distinct. We want to note that due to instrumental limitations, these measurements were done with a repetition rate of $76 \mathrm{MHz}$ leading to a build-up of photoexcited states with lifetimes longer than 13 ns. Although this build-up might impact the PL decay on longer time-scales, we expect the initial dynamics after photoexcitation to be less affected. The dynamics of the FE emission are characterized by a fast initial decay component with a decay time of around 50 ps for all particles (Figure $5 \mathrm{a}-\mathrm{c}$ ). For excitation densities above $10^{11}$ photons per $\mathrm{cm}^{2}$ an initial rise in PL intensity can be resolved for the $\mathrm{BE}$ peak, which can be fitted by a monoexponential rise function with a time constant of around 60 ps (Figure $5 \mathrm{~d}$,e). The similarity of time constants of the FE decay and the $\mathrm{BE}$ rise indicates an energy transfer from FE to BE by exciton trapping on a time-scale of tens of picoseconds. We further note that

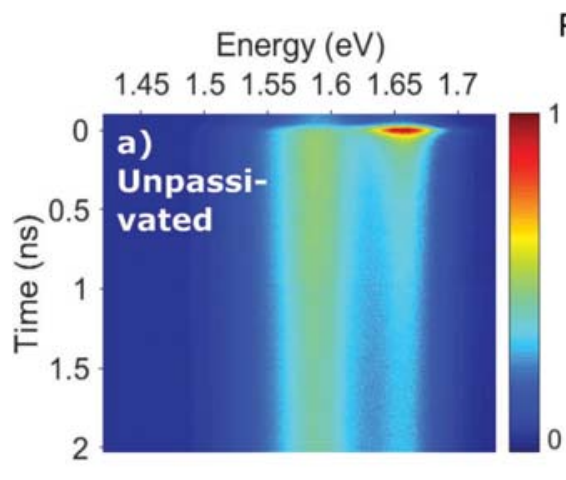

PL Intensity (a.u.)
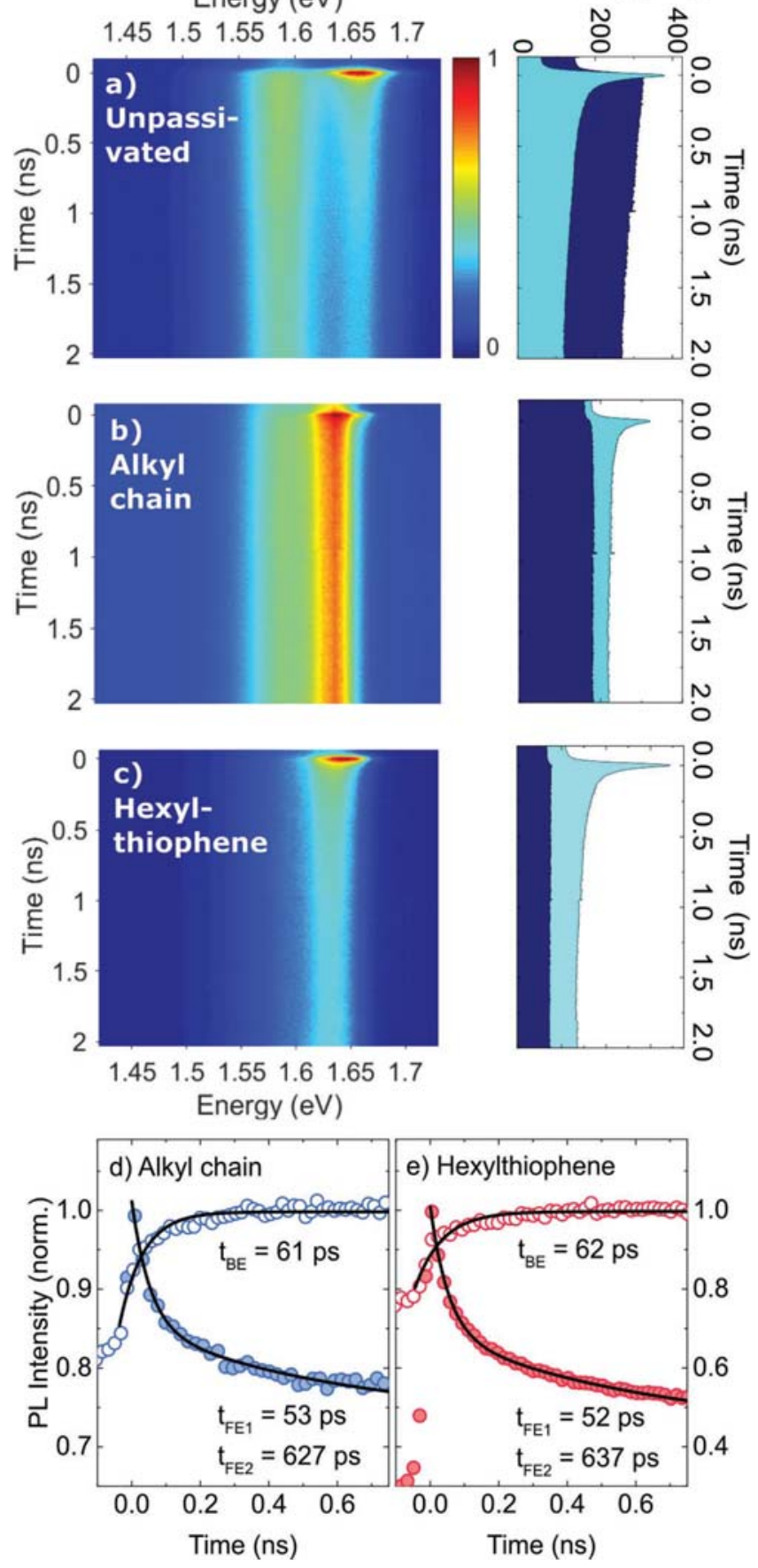

Figure 5. Fast $\mathrm{PL}$ decay components of $\mathrm{CH}_{3} \mathrm{NH}_{3} \mathrm{Pbl}_{3}$ particles measured at $50 \mathrm{~K}$ with the streak camera synchroscan unit. a-c) Left: streak camera images for excitation intensities of $7 \times 10^{10}$ photons per $\mathrm{cm}^{2}$. Right: integrated decays for free excitons (FEs) (light blue, 1.61-1.7 eV) and bound excitons (BEs) (dark blue, 1.47-1.61 eV). d,e) PL dynamics of FE (filled circles) and BE (open circles) for an excitation intensity of $2.3 \times 10^{11}$ photons per $\mathrm{cm}^{2}$, fitted with a biexponential decay function for $\mathrm{FE}$ and an exponential rise function for BE dynamics.

a comparable energy transfer mechanism from majority to minority species has been observed for tetragonal inclusions in $\mathrm{CH}_{3} \mathrm{NH}_{3} \mathrm{PbI}_{3}$ films. ${ }^{[36,50]}$ 


\subsection{Discussion of the Origin of Surface Trap States}

The PL measurements reveal a reduction of trap state energy and density as well as reduced lattice distortion for surface passivation of $\mathrm{CH}_{3} \mathrm{NH}_{3} \mathrm{PbI}_{3}$ cuboids with hexylthiophene. In contrast, surface modification with isolating alkyl chains proves to be not or much less efficient for passivation of trap states. As alkyl chain and hexylthiophene cuboids are both single-crystalline, the origin of the different trap state properties is located of the crystal surface rather than the bulk. Furthermore, low temperature PL measurements revealed identical PL peak positions of bound excitons, indicating similar trap state energies, for unpassivated particles and alkyl chain cuboids, which vary significantly in surface structure, crystallinity, and surface/volume ratio and only share a nonexistent or isolating surface passivation. Consequently, a similar origin of trap states can be assumed for unpassivated particles and alkyl chain cuboids. We therefore propose that passivation by electron-rich hexylthiophenes significantly manipulates trap state properties at the crystal surface by donating electron density to positively charged defects. This is not possible for surface passivation with isolating alkyl chains. A likely origin of these positively charged trap states is iodide vacancies at the crystal surface, as illustrated in Figure 6. The presence of positively charged surface trap states has also been proposed by Noel et al. and deQuilettes et al., who could significantly reduce nonradiative recombination and enhance solar cell performances by postdeposition of Lewis bases on $\mathrm{CH}_{3} \mathrm{NH}_{3} \mathrm{PbI}_{3}$ films. ${ }^{[27,53]}$ Similarly, PCBM-based surface treatments or aminofunctionalized electron transport layers have been applied to neutralize positively charged surface defects, improving solar cell performance and reducing the hysteresis effect. ${ }^{[29,54]}$

Despite their lower surface coverage, the successful trap state passivation of hexylthiophene cuboids demonstrates the effectiveness of passivation by electron-rich surface molecules.

Furthermore, we want to note that the facet-dependent attachment of hexylthiophene could support a more efficient trap state reduction based on a recently reported facetdependent density of trap states. ${ }^{[55]}$ The rod-like structure of the hexylthiophene cuboids might reduce the relative surface area of crystal facets possessing a higher trap state density. Likewise, it cannot be excluded that the crystal direction that is preferentially passivated by hexylthiophenes has an enhanced trap state density, enabling a more efficient passivation of surface trap states by hexylthiophenes.

\section{Conclusion}

With our model system, consisting of differently surfacepassivated single-crystalline perovskite cuboids offering high surface-to-volume ratio, we could investigate in detail the effect of surface passivation through isolating alkyl and hexylthiophene units. The impact of different crystal surface properties of $\mathrm{CH}_{3} \mathrm{NH}_{3} \mathrm{PbI}_{3}$ particles on photoexcited states at room temperature and at low temperatures has been investigated by PL measurements. Comparison of surface passivation by electron-rich hexylthiophenes and isolating alkyl chains revealed the presence of positively charged trap states at the crystal surface. The energy and density of these surface trap states can be reduced by surface passivation with hexylthiophenes, while an isolating surface passivation leads to similar trap state energies as for unpassivated surfaces. Hence, we suggest that iodide vacancies, resulting in coordinative unsaturated lead, exist at the crystal surface of $\mathrm{CH}_{3} \mathrm{NH}_{3} \mathrm{PbI}_{3}$, which act as electron traps. Yet, to further understand the impact of facet-dependent trap state passivation, the relation of crystal facets and trap state densities requires further investigation.

At room temperature, the identified surface trap states reduce the PL lifetime, increase nonradiative recombination rates, and cause surface lattice distortions. At lower temperatures, multiple PL peaks are identified to originate from bound and free excitons, whose relative population depends on the trap state properties of the particles. A fast initial PL decay component of free excitons is identified to be caused by exciton trapping on a time-scale of tens of picoseconds at $50 \mathrm{~K}$, resulting in bound excitons with lifetimes of up to several microseconds at $10 \mathrm{~K}$. As these surface trap states cause increased nonradiative recombination at room temperature, they are expected to cause losses in solar cell performance. Methods to passivate perovskite surfaces in solar cells are under current investigation, including a careful design of passivation molecules to efficiently neutralize specific crystal defects and solvent-free surface passivation strategies for solar cell fabrication. 


\section{Experimental Section}

Material Preparation: The synthesis of functionalized and unpassivated $\mathrm{CH}_{3} \mathrm{NH}_{3} \mathrm{Pbl}_{3}$ particles from the single source precursor $\mathrm{CH}_{3} \mathrm{NH}_{3} \mathrm{Pbl}_{3} \mathrm{TEG}_{2}$ (TEG = Triethylene glycol) is published elsewhere. ${ }^{[30]}$ A detailed synthesis protocol for the presented samples can be extracted from the Supporting Information. Briefly, the liquid $\mathrm{CH}_{3} \mathrm{NH}_{3} \mathrm{Pbl}_{3} \mathrm{TEG}_{2}$ was injected at $30{ }^{\circ} \mathrm{C}$ under vigorous stirring in a solution of $\mathrm{CH}_{2} \mathrm{Cl}_{2}$ containing the desired capping agent. The crystallization of $\mathrm{CH}_{3} \mathrm{NH}_{3} \mathrm{Pbl}_{3}$ was induced over time (10-15 $\mathrm{min}$ ) by selectively removing the TEG molecules. It should be mentioned that we observed a residue of TEG molecules adsorbed on the surface of all $\mathrm{CH}_{3} \mathrm{NH}_{3} \mathrm{Pbl}_{3}$ particles after the workup (see Figure S3, Supporting Information). As all particles had the same TEG on their surface and the amount of residual TEG disagreed with the trend in the density of trap states, we concluded that TEG had no influence on the observed differences in depth and density of trap states. For surface passivation, the surfactants (4-hexylthiophen2-yl)methylammonium iodide and dodecylammonium iodide had been used for hexylthiophene and alkyl chain cuboids, respectively. Silicon substrates were cleaned consecutively in detergent, acetone, and isopropanol for $5 \mathrm{~min}$, followed by $\mathrm{N}_{2}$ drying. Subsequently, the substrates were transferred to a $\mathrm{N}_{2}$ filled glovebox with moisture and oxygen levels both below $5 \mathrm{ppm}$, where the $\mathrm{CH}_{3} \mathrm{NH}_{3} \mathrm{Pbl}_{3}$ particles were dispersed in water-free chlorobenzene and dropcasted slowly at $60{ }^{\circ} \mathrm{C}$ on a hotplate.

Optical Spectroscopy: UV/Vis measurements were performed with an Agilent Cary 5000 UV-Vis-NIR spectrometer equipped with an integrating sphere. For PL measurements, samples were excited with $400 \mathrm{~nm}$ pulses at a repetition rate of $50 \mathrm{kHz}(76 \mathrm{MHz}$ for synchroscan measurements), generated by the second harmonic of a Ti:Sapphire laser (Mira 900, Coherent). An excitation density of $1.5 \times 10^{13}$ photons per $\mathrm{cm}^{2}$ was applied, if not otherwise noted. The excitation intensity was controlled by a neutral density filter. Samples were measured in vacuum in a continuous flow cryostat (Janis Research Company). The PL signal was detected by a Hamamatsu streak camera, which was equipped with a single sweep unit for long time ranges and with a synchroscan unit for time ranges $<2$ ns. For PL spectra displayed without time-resolution, full PL decays were measured with the single sweep unit and PL spectra were integrated over time. Spectral and shading corrections were applied using reference measurements with a calibrated light source. The instrument response function depended on the investigated time range and was around 12 ps for synchroscan and several nanoseconds for single sweep measurements. Low temperature measurements were done by stepwise increasing the temperature from low to high temperatures. At each temperature the sample was kept for $15 \mathrm{~min}$ before measuring.

Fitting and Data Analysis for Optical Measurements: PL spectra were fitted with three Gaussian functions. PL decays at $10 \mathrm{~K}$ were fitted using exponential functions of the form

$$
I=\sum_{n} A_{n} e^{-t / t_{n}}
$$

\section{Supporting Information}

Supporting Information is available from the Wiley Online Library or from the author.

\section{Acknowledgements}

The authors thank the Baden-Württemberg Foundation (Projects "SUPERSOL"; program "Biomimetic Materials Synthesis"), the Federal
Ministry of Education and Research (BMBF) (Project "MesoPIN") and the Carl Zeiss Foundation for financial support.

Received: September 26, 2016

Revised: November 8, 2016

Published online: December 27, 2016

[1] M. A. Green, A. Ho-Baillie, H. J. Snaith, Nat. Photonics 2014, 8, 506.

[2] A. Kojima, K. Teshima, Y. Shirai, T. Miyasaka, J. Am. Chem. Soc. 2009, 131, 6050 .

[3] W. S. Yang, J. H. Noh, N. J. Jeon, Y. C. Kim, S. Ryu, J. Seo, S. I. Seok, Science 2015, 348, 1234.

[4] S. D. Stranks, H. J. Snaith, Nat. Nanotechnol. 2015, 10, 391.

[5] N. J. Jeon, J. H. Noh, W. S. Yang, Y. C. Kim, S. Ryu, J. Seo, S. I. Seok, Nature 2015, 517, 476.

[6] F. Hao, C. C. Stoumpos, D. H. Cao, R. P. H. Chang, M. G. Kanatzidis, Nat. Photonics 2014, 8, 489.

[7] T. Leijtens, G. E. Eperon, N. K. Noel, S. N. Habisreutinger, A. Petrozza, H. J. Snaith, Adv. Energy Mater. 2015, 5, 1500963.

[8] D. Liu, M. K. Gangishetty, T. L. Kelly, J. Mater. Chem. A 2014, 2, 19873.

[9] A. Buin, P. Pietsch, J. Xu, O. Voznyy, A. H. Ip, R. Comin, E. H. Sargent, Nano Lett. 2014, 14, 6281.

[10] G. J. Wetzelaer, M. Scheepers, A. M. Sempere, C. Momblona, J. Avila, H. J. Bolink, Adv. Mater. 2015, 27, 1837.

[11] S. D. Stranks, V. M. Burlakov, T. Leijtens, J. M. Ball, A. Goriely, H. J. Snaith, Phys. Rev. Appl. 2014, 2, 034007.

[12] P. Würfel, J. Phys. C: Solid State Phys. 1982, 15, 3967.

[13] U. Rau, Phys. Rev. B. 2007, 76, 085303.

[14] W. Tress, N. Marinova, O. Inganäs, M. K. Nazeeruddin, S. M. Zakeeruddin, M. Graetzel, Adv. Energy Mater. 2015, 5, 1400812.

[15] K. Tvingstedt, O. Malinkiewicz, A. Baumann, C. Deibel, H. J. Snaith, V. Dyakonov, H. J. Bolink, Sci. Rep. 2014, 4, 6071.

[16] D. Shi, V. Adinolfi, R. Comin, M. Yuan, E. Alarousu, A. Buin, Y. Chen, S. Hoogland, A. Rothenberger, K. Katsiev, Y. Losovyj, X. Zhang, P. A. Dowben, O. F. Mohammed, E. H. Sargent, O. M. Bakr, Science 2015, 347, 519.

[17] R. E. Brandt, V. Stevanović, D. S. Ginley, T. Buonassisi, MRS Commun. 2015, 5, 265.

[18] S. Draguta, S. Thakur, Y. V. Morozov, Y. Wang, J. S. Manser, P. V. Kamat, M. Kuno, J. Phys. Chem. Lett. 2016, 7, 715.

[19] M. L. Agiorgousis, Y. Y. Sun, H. Zeng, S. Zhang, J. Am. Chem. Soc. 2014, 136, 14570.

[20] A. Baumann, S. Väth, P. Rieder, M. C. Heiber, K. Tvingstedt, V. Dyakonov, J. Phys. Chem. Lett. 2015, 6, 2350.

[21] S. D. Stranks, G. E. Eperon, G. Grancini, C. Menelaou, M. J. P. Alcocer, T. Leijtens, L. M. Herz, A. Petrozza, H. J. Snaith, Science 2013, 342, 341.

[22] W.--. Yin, H. Chen, T. Shi, S.-H. Wei, Y. Yan, Adv. Elect. Mater. 2015, 1, 1500044.

[23] J. Haruyama, K. Sodeyama, L. Han, Y. Tateyama, Acc. Chem. Res. 2016, 49, 554.

[24] J. S. Yun, A. Ho-Baillie, S. Huang, S. H. Woo, Y. Heo, J. Seidel, F. Huang, Y. B. Cheng, M. A. Green, J. Phys. Chem. Lett. 2015, 6, 875.

[25] D. W. deQuilettes, S. M. Vorpahl, S. D. Stranks, H. Nagaoka, G. E. Eperon, M. E. Ziffer, H. J. Snaith, D. S. Ginger, Science 2015, $348,683$.

[26] V. Adinolfi, M. Yuan, R. Comin, E. S. Thibau, D. Shi, M. I. Saidaminov, P. Kanjanaboos, D. Kopilovic, S. Hoogland, Z. H. Lu, O. M. Bakr, E. H. Sargent, Adv. Mater. 2016, 28, 3406.

[27] N. K. Noel, A. Abate, S. D. Stranks, E. S. Parrott, V. M. Burlakov, A. Goriely, H. J. Snaith, ACS Nano 2014, 8, 9815. 
[28] A. Abate, M. Saliba, D. J. Hollman, S. D. Stranks, K. Wojciechowski, R. Avolio, G. Grancini, A. Petrozza, H. J. Snaith, Nano Lett. 2014, 14, 3247.

[29] Y. Shao, Z. Xiao, C. Bi, Y. Yuan, J. Huang, Nat. Commun. 2014, 5, 5784

[30] T. Kollek, D. Gruber, J. Gehring, E. Zimmermann, L. Schmidt-Mende, S. Polarz, Angew. Chem., Int. Ed. Eng. 2015, 54, 1341.

[31] V. D'Innocenzo, G. Grancini, M. J. Alcocer, A. R. Kandada, S. D. Stranks, M. M. Lee, G. Lanzani, H. J. Snaith, A. Petrozza, Nat. Commun. 2014, 5, 3586.

[32] J. Even, L. Pedesseau, C. Katan, J. Phys. Chem. C. 2014, 118, 11566.

[33] L. Mao, H. Tsai, W. Nie, L. Ma, J. Im, C. C. Stoumpos, C. D. Malliakas, F. Hao, M. R. Wasielewski, A. D. Mohite, M. G. Kanatzidis, Chem. Mater. 2016, 28, 7781.

[34] C. C. Stoumpos, D. H. Cao, D. J. Clark, J. Young, J. M. Rondinelli, J. I. Jang, J. T. Hupp, M. G. Kanatzidis, Chem. Mater. 2016, 28 2852.

[35] M. T. Weller, O. J. Weber, P. F. Henry, A. M. Di Pumpo, T. C. Hansen, Chem. Commun. 2015, 51, 4180.

[36] L. Q. Phuong, Y. Yamada, M. Nagai, N. Maruyama, A. Wakamiya, Y. Kanemitsu, J. Phys. Chem. Lett. 2016, 7, 2316.

[37] Y. Yamada, T. Nakamura, M. Endo, A. Wakamiya, Y. Kanemitsu, IEEE J. Photovolt. 2015, 5, 401.

[38] A. Miyata, A. Mitioglu, P. Plochocka, O. Portugall, J. T.-W. Wang, S. D. Stranks, H. J. Snaith, R. J. Nicholas, Nat. Phys. 2015, 11, 582.

[39] Q. Lin, A. Armin, R. C. R. Nagiri, P. L. Burn, P. Meredith Nat. Photonics 2014, 9, 106

[40] H.-H. Fang, R. Raissa, M. Abdu-Aguye, S. Adjokatse, G. R. Blake, J. Even, M. A. Loi, Adv. Funct. Mater. 2015, 25, 2378.

[41] K. Wu, A. Bera, C. Ma, Y. Du, Y. Yang, L. Li, T. Wu, Phys. Chem. Chem. Phys. 2014, 16, 22476.
[42] H.-H. Fang, F. Wang, S. Adjokatse, N. Zhao, J. Even, M. Antonietta Loi, Light: Sci. Appl. 2015, 5, e16056.

[43] A. Osherov, E. M. Hutter, K. Galkowski, R. Brenes, D. K. Maude, R. J. Nicholas, P. Plochocka, V. Bulovic, T. J. Savenije, S. D. Stranks, Adv. Mater. 2016, 10.1002/adma.201604019.

[44] S. Neutzner, A. R. Srimath Kandada, G. Lanzani, A. Petrozza, J. Mater. Chem. C 2016, 4, 4630.

[45] M. I. Dar, G. Jacopin, S. Meloni, A. Mattoni, N. Arora, A. Boziki, S. M. Zakeeruddin, U. Rothlisberger, M. Graetzel, Sci. Adv. 2016, 2, 1.

[46] W. Kong, Z. Ye, Z. Qi, B. Zhang, M. Wang, A. Rahimi-Iman, H. Wu, Phys. Chem. Chem. Phys. 2015, 17, 16405.

[47] C. Wehrenfennig, M. Liu, H. J. Snaith, M. B. Johnston, L. M. Herz, APL Mater. 2014, 2, 081513

[48] X. Y. Chin, D. Cortecchia, J. Yin, A. Bruno, C. Soci, Nat. Commun $2015,6,7383$.

[49] G. Xing, N. Mathews, S. S. Lim, N. Yantara, X. Liu, D. Sabba, M. Gratzel, S. Mhaisalkar, T. C. Sum, Nat. Mater. 2014, 13, 476

[50] R. L. Milot, G. E. Eperon, H. J. Snaith, M. B. Johnston, L. M. Herz, Adv. Funct. Mater. 2015, 25, 6218

[51] J. R. Haynes, Phys. Rev. Lett. 1960, 4, 361.

[52] E. I. Rashba, G. E. Gurgenishvili, Soviet Phys.-Solid State 1962, 4, 759.

[53] D. W. deQuilettes, S. Koch, S. Burke, R. K. Paranji, A. J. Shropshire, M. E. Ziffer, D. S. Ginger, ACS Energy Lett. 2016, 1, 438.

[54] C. Sun, Z. Wu, H.-L. Yip, H. Zhang, X.-F. Jiang, Q. Xue, Z. Hu, Z. Hu, Y. Shen, M. Wang, F. Huang, Y. Cao, Adv. Energy Mater. 2016, 6, 1501534.

[55] S. Y. Leblebici, L. Leppert, Y. Li, S. E. Reyes-Lillo, S. Wickenburg, E. Wong, J. Lee, M. Melli, D. Ziegler, D. K. Angell, D. F. Ogletree, P. D. Ashby, F. M. Toma, J. B. Neaton, I. D. Sharp, A. Weber-Bargioni, Nat. Energy 2016, 1, 16093. 